\title{
Optimal Processes in a Parametric Optimal Economic Growth Model
}

\author{
Vu Thi Huong, Jen-Chih Yao and Nguyen Dong Yen*
}

\begin{abstract}
A parametric finite horizon optimal economic growth problem is solved by using the maximum principle for optimal control problems with state constraints in the book by R. Vinter [Optimal Control, Birkhäuser, Boston, 2000; Theorem 9.3.1]. From the obtained results it follows that if the total factor productivity is relatively small, then the expansion of the production facility does not lead to a higher total consumption satisfaction of the society.
\end{abstract}

\section{Introduction}

Models of economic growth have been suggested by Ramsey [25], Harrod [12], Domar [8], Solow [26], Swan [27, and others. The reader is referred to the books by Barro and Salai-Martin [3] and Acemoglu [1] for detailed information about the development of these models, which allow ones to analyze relationships among capital, labor force, production technology, and national product of an economy during a period of time.

The question of defining the consumption/saving curve to maximize a certain target of consumption satisfaction, is known as the optimal economic growth problem. It was first considered by Ramsey [25]. Later on, the problem has been extended by Cass [4] and Koopmans [18].

Necessary and sufficient optimality conditions for the solutions of optimal economic growth problems can be found in the books [28, Chapter 5], 29, Chapters 5, 7, 10, and 11], [6. Chapter 20], [1, Chapters 7 and 8], and in some papers cited therein. For infinite horizon models, some results on solution existence were given in [1, Example 7.4] and [7. Subsection 4.1]. The paper of Le Van et al. [19] establishes necessary and sufficient transversality conditions for an optimal control problem with an infinite horizon arising in dynamic models in continuous time with an application to Ramsey-like models. For finite horizon models, a few facts on solution existence are available in [7] and [24]. For a comprehensive account of this topic, we refer to a recent paper by Huong [13, which is the basis for our investigations herein. Unlike [13], here we do not discuss the solution

Received August 23, 2019; Accepted February 5, 2020.

Communicated by Jein-Shan Chen.

2010 Mathematics Subject Classification. 91B62, 49J15, 37N40, 46N10, 91B55.

Key words and phrases. optimal economic growth model, finite horizon optimal control problem, state constraint, optimal process, maximum principle.

*Corresponding author. 
existence. Our focus point is to solve the problem in question. More precisely, our aim is to give a complete synthesis of the optimal processes.

The present paper investigates a parametric finite horizon optimal economic growth problem, where the production function and the utility function are both in the form of $A K$ functions (see, e.g., [3]). By using a solution existence theorem from 13 and a maximum principle for optimal control problems with state constraints in the book by Vinter [30], we are able to prove that the problem has a unique local solution, which is also a global one, provided that the data triple satisfies a strict linear inequality. Our main theorem will be obtained via a series of nine lemmas and some involved technical arguments. Roughly speaking, we will combine an intensive treatment of the system of necessary optimality conditions given by the maximum principle with the specific properties of the given parametric optimal economic growth problem. The approach adopted herein has the origin in our preceding papers $[14,15$. From the obtained results it follows that if the total factor productivity is relatively small, then the expansion of the production facility does not lead to a higher total consumption satisfaction of the society.

The organization of the paper is as follows. Section 2 is devoted to some materials from [13] on finite horizon optimal economic growth problems. The maximum principle for optimal control problems with state constraints in [30, Theorem 9.3.1] is recalled in Section 3. Our results on optimal processes of a parametric finite horizon optimal economic growth problem, where the production function and the utility function are both in the form of AK functions, are established in Section 4.

\section{Economic growth models}

By $\mathbb{R}$ (resp., $\mathbb{R}_{+}$) we denote the set of real numbers (resp., the set of nonnegative real numbers). The Euclidean norm in the $n$-dimensional space $\mathbb{R}^{n}$ is denoted by $\|\cdot\|$. By $\mathbb{R}_{+}^{n}$ we denote the nonnegative orthant in $\mathbb{R}^{n}$. The Sobolev space $W^{1,1}\left(\left[t_{0}, T\right] ; \mathbb{R}^{n}\right.$ ) (see, e.g., [16, p. 21]) is the linear space of the absolutely continuous functions $x:\left[t_{0}, T\right] \rightarrow \mathbb{R}^{n}$ equipped with the norm

$$
\|x\|_{W^{1,1}}=\left\|x\left(t_{0}\right)\right\|+\int_{t_{0}}^{T}\|\dot{x}(t)\| d t .
$$

We now recall some materials from Subsection 2.1 of the paper by Huong [13], and refer the interested reader to the books [28, Chapter 5], 29, Chapters 5, 7, 10, and 11], 6. Chapter 20], 1. Chapters 7 and 8] for systematical presentations of optimal economic growth models.

Consider the problem of optimal growth of an aggregative economy. The national product at time $t$ is denoted $Y(t)$. Suppose that the dependence of $Y(t)$ on the labor $L(t)$ 
and the capital $K(t)$ is described by $Y(t)=F(K(t), L(t))$ with $t \geq 0$, where $F: \mathbb{R}_{+}^{2} \rightarrow \mathbb{R}_{+}$ is the production function. It is assumed that

$$
F(\alpha K, \alpha L)=\alpha F(K, L)
$$

for any $(K, L) \in \mathbb{R}_{+}^{2}$ and $\alpha>0$. For every $t \geq 0$, by $C(t)$ and $I(t)$, respectively, we denote the consumption amount and the investment amount of the economy. The equilibrium relation in the output market is depicted by

$$
Y(t)=C(t)+I(t), \quad \forall t \geq 0 .
$$

The relationship between the capital $K(t)$ and the investment amount $I(t)$ is given by the differential equation

$$
\dot{K}(t)=I(t), \quad \forall t \geq 0
$$

where $\dot{K}(t)=\frac{d K(t)}{d t}$ denotes the Fréchet derivative of $K(\cdot)$ at time instance $t$ (see, e.g., 6 , pp. 465-466]). If the investment function $I(\cdot)$ is continuous, then one can compute the capital stock $K(t)$ at time $t$ by the formula

$$
K(t)=K(0)+\int_{0}^{t} I(\tau) d \tau,
$$

where the integral is Riemannian and $K(0)$ signifies the initial capital stock. In particular, the rate of increase of the capital stock $\dot{K}(t)$ at every time moment $t$ exists and it is finite. If the initial labor amount is $L_{0}>0$ and the rate of labor force is a constant $\sigma>0$ (i.e., $\dot{L}(t)=\sigma L(t)$ for all $t \geq 0)$, then the labor amount at time moment $t$ is

$$
L(t)=L_{0} e^{\sigma t}, \quad \forall t \geq 0 .
$$

For any $t \geq 0$, as $L(t)>0$, from $(2.2)$ we have

$$
\frac{Y(t)}{L(t)}=F\left(\frac{K(t)}{L(t)}, 1\right), \quad \forall t \geq 0
$$

By considering the capital-to-labor ratio $k(t):=\frac{K(t)}{L(t)}$ and the function $\phi(k):=F(k, 1)$ for $k \geq 0$, from the last equality we have

$$
\phi(k(t))=\frac{Y(t)}{L(t)}, \quad \forall t \geq 0
$$

Due to (2.6), one calls $\phi(k(t))$ the output per capita at time $t$ and $\phi(\cdot)$ the per capita production function. Since $F$ has nonnegative values, so does $\phi$. Combining the continuous differentiability of $K(\cdot)$ and $L(\cdot)$, which is guaranteed by (2.4) and (2.5), with the equality 
defining the capital-to-labor ratio, one can assert that $k(\cdot)$ is continuously differentiable. Thus, from the relation $K(t)=k(t) L(t)$ one obtains

$$
\dot{K}(t)=\dot{k}(t) L(t)+k(t) \dot{L}(t), \quad \forall t \geq 0 .
$$

Dividing both sides of the above equality by $L(t)$ and recalling that $\dot{L}(t)=\sigma L(t)$, we get

$$
\frac{\dot{K}(t)}{L(t)}=\dot{k}(t)+\sigma k(t), \quad \forall t \geq 0 .
$$

Similarly, dividing both sides of the equality in $(2.4)$ by $L(t)$ and using (2.3), we have

$$
\frac{\dot{K}(t)}{L(t)}=\frac{Y(t)}{L(t)}-\frac{C(t)}{L(t)}, \quad \forall t \geq 0 .
$$

So, by considering the per capita consumption $c(t):=\frac{C(t)}{L(t)}$ of the economy at time $t$ and invoking 2.6), one obtains

$$
\frac{\dot{K}(t)}{L(t)}=\phi(k(t))-c(t), \quad \forall t \geq 0 .
$$

Combining this with 2.7 yields

$$
\dot{k}(t)=\phi(k(t))-\sigma k(t)-c(t), \quad \forall t \geq 0
$$

The amount of consumption at time $t$ is

$$
C(t)=(1-s(t)) Y(t), \quad \forall t \geq 0
$$

with $s(t) \in[0,1]$ being the propensity to save at time $t$ (thus, $1-s(t)$ is the propensity to consume at time $t)$. Then, by dividing both sides of $(2.9)$ by $L(t)$ and referring to (2.6), one gets

$$
c(t)=(1-s(t)) \phi(k(t)), \quad \forall t \geq 0 .
$$

Thanks to 2.10, one can rewrite 2.8 equivalently as

$$
\dot{k}(t)=s(t) \phi(k(t))-\sigma k(t), \quad \forall t \geq 0 .
$$

In the special case where $s(\cdot)$ is a constant function, i.e., $s(t)=s>0$ for all $t \geq 0$, relation 2.11) is the fundamental equation of the neo-classical aggregate growth model of Solow 26].

One major concern of the planners is to choose a pair of functions $(k, c)$ (or $(k, s))$ defined on a planning interval $\left[t_{0}, T\right] \subset[0,+\infty]$, that satisfies (2.8) (or (2.11) ) and the initial condition $k\left(t_{0}\right)=k_{0}$, to maximize a certain target of consumption. Here $k_{0}>0$ is a 
given value. As the target function one may choose is $\int_{t_{0}}^{T} c(t) d t$, which is the total amount of per capita consumption on the time period $\left[t_{0}, T\right]$. A more general kind of the target function is $\int_{t_{0}}^{T} \omega(c(t)) e^{-\lambda t} d t$, where $\omega: \mathbb{R}_{+} \rightarrow \mathbb{R}$ is a utility function associated with the representative individual consumption $c(t)$ in the society, $\lambda \geq 0$ is called the real interest rate, and $e^{-\lambda t}$ is the time discount factor. The just mentioned planning task is an optimal control problem. Interpreting $k(t)$ as the state trajectory and $s(t)$ as the control function, one can formulate the problem as follows.

Let there be given a production function $F: \mathbb{R}_{+}^{2} \rightarrow \mathbb{R}_{+}$satisfying $(2.2)$ for any $(K, L)$ from $\mathbb{R}_{+}^{2}$ and $\alpha>0$. Define the function $\phi(k)$ on $\mathbb{R}_{+}$by setting $\phi(k)=F(k, 1)$. Assume that a finite time interval $\left[t_{0}, T\right]$ with $T>t_{0} \geq 0$, a utility function $\omega: \mathbb{R}_{+} \rightarrow \mathbb{R}$, and a real interest rate $\lambda \geq 0$ are given. Since $c(t)=(1-s(t)) \phi(k(t))$ by $(2.10)$, the target function can be expressed via $k(t)$ and $s(t)$ as

$$
\int_{t_{0}}^{T} \omega(c(t)) e^{-\lambda t} d t=\int_{t_{0}}^{T} \omega[(1-s(t)) \phi(k(t))] e^{-\lambda t} d t .
$$

So, the problem of finding an optimal growth process for an aggregative economy is the following one:

$$
\text { Maximize } I(k, s):=\int_{t_{0}}^{T} \omega[(1-s(t)) \phi(k(t))] e^{-\lambda t} d t
$$

over $k \in W^{1,1}\left(\left[t_{0}, T\right] ; \mathbb{R}\right)$ and measurable functions $s:\left[t_{0}, T\right] \rightarrow \mathbb{R}$ satisfying

$$
\begin{cases}\dot{k}(t)=s(t) \phi(k(t))-\sigma k(t) & \text { a.e. } t \in\left[t_{0}, T\right], \\ k\left(t_{0}\right)=k_{0}, & \text { a.e. } t \in\left[t_{0}, T\right], \\ s(t) \in[0,1] & \forall t \in\left[t_{0}, T\right] .\end{cases}
$$

This problem has five parameters: $\lambda \geq 0, \sigma>0, k_{0}>0, t_{0}, T$.

The optimal control problem in 2.12 and 2.13 will be denoted by (GP). According to [5], (GP) is a finite horizon optimal control problem of the Lagrange type. Recall that a function $\varphi:\left[t_{0}, T\right] \rightarrow \mathbb{R}$ is said to be measurable if for any $\alpha \in \mathbb{R}$ the set $\left\{t \in\left[t_{0}, T\right] \mid \varphi \in\right.$ $(-\infty, \alpha)\}$ is Lebesgue measurable. This amounts to saying that, for every Borel set $B$ in $\mathbb{R}$, the inverse set $\varphi^{-1}(B):=\left\{t \in\left[t_{0}, T\right] \mid \varphi \in B\right\}$ is Lebesgue measurable.

For each $\alpha, \beta \in(0,1]$, let $\phi(k)=A k^{\alpha}$ for all $k \geq 0$ and $\omega(c)=c^{\beta}$ for every $c \geq 0$ with $A>0$ being a constant in (GP). Then, we have the optimal control problem

$$
\text { Maximize } \int_{t_{0}}^{T}[1-s(t)]^{\beta} k^{\alpha \beta}(t) e^{-\lambda t} d t
$$


over $k \in W^{1,1}\left(\left[t_{0}, T\right] ; \mathbb{R}\right)$ and measurable functions $s:\left[t_{0}, T\right] \rightarrow \mathbb{R}$ satisfying

$$
\begin{cases}\dot{k}(t)=A k^{\alpha}(t) s(t)-\sigma k(t) & \text { a.e. } t \in\left[t_{0}, T\right], \\ k\left(t_{0}\right)=k_{0}, & \text { a.e. } t \in\left[t_{0}, T\right], \\ s(t) \in[0,1] & \forall t \in\left[t_{0}, T\right] \\ k(t) \geq 0 & \end{cases}
$$

with $\lambda \geq 0, A>0, \sigma>0, k_{0}>0$, and $T>t_{0} \geq 0$ being given parameters. We will denote the problem in (2.14) and 2.15) by $\left(\mathrm{GP}_{1}\right)$. According to [5], $\left(\mathrm{GP}_{1}\right)$ is a finite horizon optimal control problem of the Lagrange type.

The next solution existence theorem for $\left(\mathrm{GP}_{1}\right)$ will be useful for our further investigations.

Theorem 2.1. [13, Theorem 4.1] For any constants $\alpha \in(0,1]$ and $\beta \in(0,1]$, the optimal economic growth problem $\left(\mathrm{GP}_{1}\right)$ possesses a global solution.

3. Maximum principle for optimal control problems with state constraints

Let $\Omega \subset \mathbb{R}^{n}$ be a closed set and $\bar{v} \in \Omega$. The Fréchet normal cone (also called the prenormal cone, or the regular normal cone) to $\Omega$ at $\bar{v}$ is given by

$$
\widehat{N}_{\Omega}(\bar{v})=\left\{\begin{array}{l|l}
v^{\prime} \in \mathbb{R}^{n} \mid \limsup _{\substack{\Omega \\
\lim ^{\prime}}} \frac{\left\langle v^{\prime}, v-\bar{v}\right\rangle}{\|v-\bar{v}\|} \leq 0
\end{array}\right\},
$$

where $v \stackrel{\Omega}{\rightarrow} \bar{v}$ means $v \rightarrow \bar{v}$ with $v \in \Omega$. The Mordukhovich (or limiting) normal cone to $\Omega$ at $\bar{v}$ is defined by

$$
N_{\Omega}(\bar{v})=\left\{v^{\prime} \in \mathbb{R}^{n} \mid \exists \text { sequences } v_{k} \rightarrow \bar{v}, v_{k}^{\prime} \rightarrow v^{\prime} \text { with } v_{k}^{\prime} \in \widehat{N}_{\Omega}\left(v_{k}\right) \text { for all } k \in \mathbb{N}\right\} .
$$

Given an extended real-valued function $\varphi: \mathbb{R}^{n} \rightarrow \mathbb{R} \cup\{-\infty,+\infty\}$, one defines the epigraph of $\varphi$ by epi $\varphi=\left\{(x, \mu) \in \mathbb{R}^{n} \times \mathbb{R} \mid \mu \geq \varphi(x)\right\}$. The Mordukhovich subdifferential (or limiting subdifferential) of $\varphi$ at $\bar{x} \in \mathbb{R}^{n}$ with $|\varphi(\bar{x})|<\infty$ is defined by

$$
\partial \varphi(\bar{x})=\left\{x^{*} \in \mathbb{R}^{n} \mid\left(x^{*},-1\right) \in N_{\operatorname{epi} \varphi}((\bar{x}, \varphi(\bar{x})))\right\} .
$$

If $|\varphi(x)|=\infty$, then one puts $\partial \varphi(\bar{x})=\emptyset$. The reader is referred to $21-23$ for comprehensive treatments of the Fréchet normal cone, the limiting normal cone, the limiting subdifferential, and the related calculus rules.

As in [30, p. 321], we consider the following finite horizon optimal control problem of the Mayer type, denoted by $\mathcal{M}$,

$$
\text { Minimize } g\left(x\left(t_{0}\right), x(T)\right)
$$


over $x \in W^{1,1}\left(\left[t_{0}, T\right] ; \mathbb{R}^{n}\right)$ and measurable functions $u:\left[t_{0}, T\right] \rightarrow \mathbb{R}^{m}$ satisfying

$$
\begin{cases}\dot{x}(t)=f(t, x(t), u(t)) & \text { a.e. } t \in\left[t_{0}, T\right], \\ \left(x\left(t_{0}\right), x(T)\right) \in C, & \\ u(t) \in U(t) & \text { a.e. } t \in\left[t_{0}, T\right], \\ h(t, x(t)) \leq 0 & \forall t \in\left[t_{0}, T\right],\end{cases}
$$

where $\left[t_{0}, T\right]$ is a given interval, $g: \mathbb{R}^{n} \times \mathbb{R}^{n} \rightarrow \mathbb{R}, f:\left[t_{0}, T\right] \times \mathbb{R}^{n} \times \mathbb{R}^{m} \rightarrow \mathbb{R}^{n}$, and $h:\left[t_{0}, T\right] \times \mathbb{R}^{n} \rightarrow \mathbb{R}$ are given functions, $C \subset \mathbb{R}^{n} \times \mathbb{R}^{n}$ is a closed set, and $U:\left[t_{0}, T\right] \rightrightarrows \mathbb{R}^{m}$ is a set-valued map.

A measurable function $u:\left[t_{0}, T\right] \rightarrow \mathbb{R}^{m}$ satisfying $u(t) \in U(t)$ a.e. $t \in\left[t_{0}, T\right]$ is called a control function. A process $(x, u)$ consists of a control function $u$ and an arc $x \in$ $W^{1,1}\left(\left[t_{0}, T\right] ; \mathbb{R}^{n}\right)$ that is a solution to the differential equation in 3.2 . A state trajectory $x$ is the first component of some process $(x, u)$. A process $(x, u)$ is called feasible if the state trajectory satisfies the endpoint constraint $\left(x\left(t_{0}\right), x(T)\right) \in C$ and the state constraint $h(t, x(t)) \leq 0$ for all $t \in\left[t_{0}, T\right]$.

Due to the appearance of the state constraint $h(t, x(t)) \geq 0, t \in\left[t_{0}, T\right]$, the problem $\mathcal{M}$ in (3.1) and (3.2) is said to be an optimal control problem with state constraints. But, if the inequality $h(t, x(t)) \leq 0$ is fulfilled for every $(t, x(t))$ with $t \in\left[t_{0}, T\right]$ and $x \in W^{1,1}\left(\left[t_{0}, T\right] ; \mathbb{R}^{n}\right)$ (for example, when $h$ is constant function having a fixed nonpositive value), i.e., the condition $h(t, x(t)) \leq 0$ for all $t \in\left[t_{0}, T\right]$ can be removed from (3.2), then one says that $\mathcal{M}$ an optimal control problem without state constraints.

The convex hull of a subset $C \subset \mathbb{R}^{n}$ is denoted by co $C$. The graph of a set-valued map $F: \mathbb{R}^{n} \rightrightarrows \mathbb{R}^{m}$ is defined by gph $F:=\left\{(x, y) \in \mathbb{R}^{n} \times \mathbb{R}^{m} \mid y \in F(x)\right\}$. For a given segment $\left[t_{0}, T\right]$ of the real line, we denote the $\sigma$-algebra of its Lebesgue measurable subsets (resp., the $\sigma$-algebra of its Borel measurable subsets) by $\mathcal{L}$ (resp., $\mathcal{B}$ ).

The Hamiltonian $\mathcal{H}:\left[t_{0}, T\right] \times \mathbb{R}^{n} \times \mathbb{R}^{n} \times \mathbb{R}^{m} \rightarrow \mathbb{R}$ of $(3.2)$ is defined by

$$
\mathcal{H}(t, x, p, u):=p \cdot f(t, x, u)=\sum_{i=1}^{n} p_{i} f_{i}(t, x, u) .
$$

Definition 3.1. A feasible process $(\bar{x}, \bar{u})$ is called a $W^{1,1}$ local minimizer for $\mathcal{M}$ if there exists $\delta>0$ such that $g\left(\bar{x}\left(t_{0}\right), \bar{x}(T)\right) \leq g\left(x\left(t_{0}\right), x(T)\right)$ for any feasible processes $(x, u)$ satisfying $\|\bar{x}-x\|_{W^{1,1}} \leq \delta$.

Definition 3.2. A feasible process $(\bar{x}, \bar{u})$ is called a $W^{1,1}$ global minimizer for $\mathcal{M}$ if, for any feasible processes $(x, u)$, one has $g\left(\bar{x}\left(t_{0}\right), \bar{x}(T)\right) \leq g\left(x\left(t_{0}\right), x(T)\right)$.

Definition 3.3. [30, p. 329] The partial hybrid subdifferential $\partial_{x}^{>} h(t, x)$ of $h(t, x)$ w.r.t. $x$ 
is given by

$$
\begin{aligned}
& \partial_{x}^{>} h(t, x):=\operatorname{co}\left\{\xi \mid \text { there exists }\left(t_{i}, x_{i}\right) \stackrel{h}{\rightarrow}(t, x)\right. \text { such that } \\
& \left.\qquad h\left(t_{k}, x_{k}\right)>0 \text { for all } k \text { and } \nabla_{x} h\left(t_{k}, x_{k}\right) \rightarrow \xi\right\},
\end{aligned}
$$

where the symbol $\left(t_{k}, x_{k}\right) \stackrel{h}{\rightarrow}(t, x)$ means that $\left(t_{k}, x_{k}\right) \rightarrow(t, x)$ and $h\left(t_{k}, x_{k}\right) \rightarrow h(t, x)$ as $k \rightarrow \infty$.

To deal with the state constraint $h(t, x(t)) \leq 0$ in $\mathcal{M}$, one introduces a multiplier that is an element in the topological dual $C^{*}\left(\left[t_{0}, T\right] ; \mathbb{R}\right)$ of the space of continuous functions $C\left(\left[t_{0}, T\right] ; \mathbb{R}\right)$ with the supremum norm. By the Riesz Representation Theorem (see, e.g., [17, Theorem 6, p. 374] and [20, Theorem 1, pp. 113-115]), any bounded linear functional $f$ on $C\left(\left[t_{0}, T\right] ; \mathbb{R}\right)$ can be uniquely represented in the form

$$
f(x)=\int_{\left[t_{0}, T\right]} x(t) d v(t),
$$

where $v$ is a function of bounded variation on $\left[t_{0}, T\right]$ which vanishes at $t_{0}$ and which are continuous from the right at every point $\tau \in\left(t_{0}, T\right)$, and $\int_{\left[t_{0}, T\right]} x(t) d v(t)$ is the RiemannStieltjes integral of $x$ with respect to $v$ (see, e.g., [17, p. 364]). The set of the elements of $C^{*}\left(\left[t_{0}, T\right] ; \mathbb{R}\right)$ which are given by nondecreasing functions $v$ is denoted by $C^{\oplus}\left(t_{0}, T\right)$.

Every $v \in C^{*}\left(\left[t_{0}, T\right] ; \mathbb{R}\right)$ corresponds to a finite regular measure, denoted by $\mu_{v}$, on the $\sigma$-algebra $\mathcal{B}$ of the Borel subsets of $\left[t_{0}, T\right]$ by the formula

$$
\mu_{v}(A):=\int_{\left[t_{0}, T\right]} \chi_{A}(t) d v(t),
$$

where $\chi_{A}(t)=1$ for $t \in A$ and $\chi_{A}(t)=0$ if $t \notin A$. Due to the correspondence $v \mapsto \mu_{v}$, we call every element $v \in C^{*}\left(\left[t_{0}, T\right] ; \mathbb{R}\right)$ a "measure" and identify $v$ with $\mu_{v}$. Clearly, the measure corresponding to each $v \in C^{\oplus}\left(t_{0}, T\right)$ is nonnegative.

The integrals $\int_{\left[t_{0}, t\right)} \nu(s) d \mu(s)$ and $\int_{\left[t_{0}, T\right]} \nu(s) d \mu(s)$ of a Borel measurable function $\nu$ in next theorem are understood in the sense of the Lebesgue-Stieltjes integration [17, p. 364].

Theorem 3.4. 30, Theorem 9.3.1] Let $(\bar{x}, \bar{u})$ be a $W^{1,1}$ local minimizer for $\mathcal{M}$. Assume that for some $\delta>0$, the following hypotheses are satisfied:

(H1) $f(\cdot, x, \cdot)$ is $\mathcal{L} \times \mathcal{B}^{m}$ measurable, for fixed $x$. There exists a Borel measurable function $k(\cdot, \cdot):\left[t_{0}, T\right] \times \mathbb{R}^{m} \rightarrow \mathbb{R}$ such that $t \mapsto k(t, \bar{u}(t))$ is integrable and

$$
\left\|f(t, x, u)-f\left(t, x^{\prime}, u\right)\right\| \leq k(t, u)\left\|x-x^{\prime}\right\|, \quad \forall x, x^{\prime} \in \bar{x}(t)+\delta \bar{B}, \forall u \in U(t)
$$

for almost every $t \in\left[t_{0}, T\right]$;

(H2) gph $U$ is a Borel set in $\left[t_{0}, T\right] \times \mathbb{R}^{m}$; 
(H3) $g$ is Lipschitz continuous on the ball $\left(\bar{x}\left(t_{0}\right), \bar{x}(T)\right)+\delta \bar{B}$;

(H4) $h$ is upper semicontinuous and there exists $K>0$ such that

$$
\left\|h(t, x)-h\left(t, x^{\prime}\right)\right\| \leq K\left\|x-x^{\prime}\right\|, \quad \forall x, x^{\prime} \in \bar{x}(t)+\delta \bar{B}, \forall t \in\left[t_{0}, T\right] .
$$

Then there exist $p \in W^{1,1}\left(\left[t_{0}, T\right] ; \mathbb{R}^{n}\right), \gamma \geq 0, \mu \in C^{\oplus}\left(t_{0}, T\right)$, and a Borel measurable function $\nu:\left[t_{0}, T\right] \rightarrow \mathbb{R}^{n}$ such that $(p, \mu, \gamma) \neq(0,0,0)$, and for $q(t):=p(t)+\eta(t)$ with $\eta(t):=\int_{\left[t_{0}, t\right)} \nu(s) d \mu(s)$ if $t \in\left[t_{0}, T\right)$ and $\eta(T):=\int_{\left[t_{0}, T\right]} \nu(s) d \mu(s)$, the following holds true:

(i) $\nu(t) \in \partial_{x}^{>} h(t, \bar{x}(t)) \mu-a . e$;

(ii) $-\dot{p}(t) \in \operatorname{co} \partial_{x} \mathcal{H}(t, \bar{x}(t), q(t), \bar{u}(t))$ a.e.;

(iii) $\left(p\left(t_{0}\right),-q(T)\right) \in \gamma \partial g\left(\bar{x}\left(t_{0}\right), \bar{x}(T)\right)+N_{C}\left(\bar{x}\left(t_{0}\right), \bar{x}(T)\right)$;

(iv) $\mathcal{H}(t, \bar{x}(t), q(t), \bar{u}(t))=\max _{u \in U(t)} \mathcal{H}(t, \bar{x}(t), q(t), u)$ a.e.

\section{Finding the optimal processes}

To apply Theorem 3.4 for finding optimal processes for $\left(\mathrm{GP}_{1}\right)$, we have to interpret $\left(\mathrm{GP}_{1}\right)$ in the form of $\mathcal{M}$. For doing so, we set $x(t)=\left(x_{1}(t), x_{2}(t)\right)$, where $x_{1}(t)$ plays the role of $k(t)$ in 2.14, 2.15) and

$$
x_{2}(t):=-\int_{t_{0}}^{t}[1-s(\tau)]^{\beta} x_{1}^{\alpha \beta}(\tau) e^{-\lambda \tau} d \tau
$$

for all $t \in[0, T]$. Thus, $\left(\mathrm{GP}_{1}\right)$ is equivalent to the following problem:

$$
\text { Minimize } x_{2}(T)
$$

over $x=\left(x_{1}, x_{2}\right) \in W^{1,1}\left(\left[t_{0}, T\right] ; \mathbb{R}^{2}\right)$ and measurable functions $s:\left[t_{0}, T\right] \rightarrow \mathbb{R}$ satisfying

$$
\begin{cases}\dot{x}_{1}(t)=A x_{1}^{\alpha}(t) s(t)-\sigma x_{1}(t) & \text { a.e. } t \in\left[t_{0}, T\right], \\ \dot{x}_{2}(t)=-[1-s(t)]^{\beta} x_{1}^{\alpha \beta}(t) e^{-\lambda t} & \text { a.e. } t \in\left[t_{0}, T\right], \\ \left(x\left(t_{0}\right), x(T)\right) \in\left\{\left(k_{0}, 0\right)\right\} \times \mathbb{R}^{2}, & \\ s(t) \in[0,1] & \text { a.e. } t \in\left[t_{0}, T\right], \\ x_{1}(t) \geq 0 & \forall t \in\left[t_{0}, T\right] .\end{cases}
$$

The optimal control problem with state constraints in 4.2 and 4.3 is denoted by $\left(\mathrm{GP}_{1 a}\right)$.

To see $\left(\mathrm{GP}_{1 a}\right)$ in the form of $\mathcal{M}$, one chooses $n=m=1, C=\left\{\left(k_{0}, 0\right)\right\} \times \mathbb{R}^{2}, U(t)=$ $[0,1]$ for $t \in\left[t_{0}, T\right], g(x, y)=y_{2}$ for $x=\left(x_{1}, x_{2}\right) \in \mathbb{R}^{2}$ and $y=\left(y_{1}, y_{2}\right) \in \mathbb{R}^{2}, h(t, x)=-x_{1}$ 
for $(t, x) \in\left[t_{0}, T\right] \times \mathbb{R}^{2}$. As concerning the function $f$, for any $(t, x, s) \in\left[t_{0}, T\right] \times \mathbb{R}^{2} \times \mathbb{R}$, one lets $f(t, x, s)=\left(A x_{1}^{\alpha} s-\sigma x_{1},-(1-s)^{\beta} x_{1}^{\alpha \beta} e^{-\lambda t}\right)$ if $x_{1} \in \mathbb{R}_{+}$and $s \in[0,1]$, and defines $f(t, x, s)$ in a suitable way if $x_{1} \notin \mathbb{R}_{+}$, or $s \notin[0,1]$.

Let $(\bar{x}, \bar{s})$ be a $W^{1,1}$ local minimizer for $\left(\mathrm{GP}_{1 a}\right)$. To satisfy the assumption $(\mathrm{H} 1)$ in Theorem 3.4. for any $s \in[0,1]$, the function $f(t, \cdot, s)$ must be locally Lipschitz around $\bar{x}(t)$ for almost every $t \in\left[t_{0}, T\right]$. This requirement cannot be satisfied if $\alpha \in(0,1)$ and the set of $t \in\left[t_{0}, T\right]$ when the curve $\bar{x}_{1}(t)$ hits the lower bound $x_{1}=0$ of the state constraint $x_{1}(t) \geq 0$ has a positive measure. To overcome this situation, we may use one of the following two additional assumptions:

(A1) $\alpha=1$

(A2) $\alpha \in(0,1)$ and the set $\left\{t \in\left[t_{0}, T\right] \mid \bar{x}_{1}(t)=0\right\}$ has the Lebesgue measure 0, i.e., $\bar{x}_{1}(t)>0$ for almost every $t \in\left[t_{0}, T\right]$.

Regarding the exponent $\beta \in(0,1]$, we distinguish two cases: $(\mathrm{B} 1) \beta=1 ;(\mathrm{B} 2) \beta \in(0,1)$.

From now on, we will consider problem $\left(\mathrm{GP}_{1 a}\right)$ under the conditions $(\mathrm{A} 1)$ and (B1). Thanks to these assumptions, we have

$$
f(t, x, s)=\left(A x_{1}^{\alpha} s-\sigma x_{1},-(1-s)^{\beta} x_{1}^{\alpha \beta} e^{-\lambda t}\right)=\left((A s-\sigma) x_{1},(s-1) x_{1} e^{-\lambda t}\right)
$$

if $x_{1} \in \mathbb{R}_{+}$and $s \in[0,1]$. Clearly, the most natural extension of the function $f$ from the domain $\left[t_{0}, T\right] \times \mathbb{R}_{+} \times \mathbb{R} \times[0,1]$ to $\left[t_{0}, T\right] \times \mathbb{R}^{2} \times \mathbb{R}$, which is the domain of variables required by Theorem 3.4 , is as follows:

$$
f(t, x, s)=\left((A s-\sigma) x_{1},(s-1) x_{1} e^{-\lambda t}\right), \quad \forall(t, x, s) \in\left[t_{0}, T\right] \times \mathbb{R}^{2} \times \mathbb{R} .
$$

In accordance with (3.3) and (4.4), the Hamiltonian of $\left(\mathrm{GP}_{1 a}\right)$ is given by

$$
\mathcal{H}(t, x, p, s)=(A s-\sigma) x_{1} p_{1}+(s-1) x_{1} e^{-\lambda t} p_{2}
$$

for every $(t, x, p, s) \in\left[t_{0}, T\right] \times \mathbb{R}^{2} \times \mathbb{R}^{2} \times \mathbb{R}$. Since the function in (4.5) is continuously differentiable in $x$, we have

$$
\partial_{x} \mathcal{H}(t, x, p, u)=\left\{\left((A s-\sigma) p_{1}+(s-1) e^{-\lambda t} p_{2}, 0\right)\right\}
$$

for all $(t, x, p, s) \in\left[t_{0}, T\right] \times \mathbb{R}^{2} \times \mathbb{R}^{2} \times \mathbb{R}$. By (3.4), the partial hybrid subdifferential of $h$ at $(t, x) \in\left[t_{0}, T\right] \times \mathbb{R}^{2}$ is given by

$$
\partial_{x}^{>} h(t, x)= \begin{cases}\emptyset & \text { if } x_{1}>0 \\ \{(-1,0)\} & \text { if } x_{1} \leq 0\end{cases}
$$

The relationships between a control function $s(\cdot)$ and the corresponding trajectory $x(\cdot)$ of 4.3 can be described as follows. 
Lemma 4.1. For each measurable function $s:\left[t_{0}, T\right] \rightarrow \mathbb{R}$ with $s(t) \in[0,1]$, there exists a unique trajectory $x=\left(x_{1}, x_{2}\right) \in W^{1,1}\left(\left[t_{0}, T\right] ; \mathbb{R}^{2}\right)$ such that $(x, s)$ is a feasible process of (4.3). Moreover, for every $\tau \in\left[t_{0}, T\right]$, one has

$$
x_{1}(t)=x_{1}(\tau) e^{\int_{\tau}^{t}(A s(z)-\sigma) d z}, \quad \forall t \in\left[t_{0}, T\right] .
$$

In particular, $x_{1}(t)>0$ for all $t \in\left[t_{0}, T\right]$.

Proof. Given a function $s$ satisfying the assumptions of the lemma, we suppose that $x=\left(x_{1}, x_{2}\right) \in W^{1,1}\left(\left[t_{0}, T\right] ; \mathbb{R}^{2}\right)$ such that $(x, s)$ is a feasible process of (4.3). Then, the condition $\alpha=1$ implies that

$$
\left\{\begin{array}{l}
\dot{x}_{1}(t)=[A s(t)-\sigma] x_{1}(t) \quad \text { a.e. } t \in\left[t_{0}, T\right], \\
x_{1}\left(t_{0}\right)=k_{0} .
\end{array}\right.
$$

Since $s(\cdot)$ is measurable and bounded on $\left[t_{0}, T\right]$, so is the function $t \mapsto A s(t)-\sigma$. In particular, the latter is Lebesgue integrable on $\left[t_{0}, T\right]$. Hence, by the lemma in $[2$, pp. $121-$ 122 ] on the solution existence and uniqueness of the Cauchy problem for linear differential equations, one knows that 4.9 has a unique solution. Thus, $x_{1}(\cdot)$ is defined uniquely via $s(\cdot)$. This and the equality $x_{2}(t)=-\int_{t_{0}}^{t}[1-s(\tau)] x_{1}(\tau) e^{-\lambda \tau} d \tau$, which follows from 4.1 together with the conditions $\alpha=1$ and $\beta=1$, imply the uniqueness of $x_{2}(\cdot)$. To prove the second assertion, put

$$
\Omega(t, \tau)=e^{\int_{\tau}^{t}(A s(z)-\sigma) d z}, \quad \forall t, \tau \in\left[t_{0}, T\right]
$$

By the Lebesgue integrability of the function $t \mapsto A s(t)-\sigma$ on $\left[t_{0}, T\right], \Omega(t, \tau)$ is well defined on $\left[t_{0}, T\right] \times\left[t_{0}, T\right]$, and by $[17$, Theorem 8 , p. 324] one has

$$
\frac{d}{d t}\left(\int_{\tau}^{t}(A s(z)-\sigma) d z\right)=A s(t)-\sigma \quad \text { a.e. } t \in\left[t_{0}, T\right] .
$$

Therefore, from 4.10 and 4.11) it follows that $\Omega(\cdot, \tau)$ is the solution of the Cauchy problem

$$
\frac{d}{d t} \Omega(t, \tau)=(A s(t)-\sigma) \Omega(t, \tau) \text { a.e. } t \in\left[t_{0}, T\right] \quad \text { and } \quad \Omega(\tau, \tau)=1
$$

In other words, the real-valued function $\Omega(t, \tau)$ of the variables $t$ and $\tau$ is the principal matrix solution (see [2, p. 123]) specialized to the homogeneous differential equation in 4.9. Hence, by the theorem in [2, p. 123] on the solution of linear differential equations, we obtain (4.8). Since $x_{1}\left(t_{0}\right)=k_{0}>0$, applying (4.8) for $\tau=t_{0}$ implies that $x_{1}(t)>0$ for all $t \in\left[t_{0}, T\right]$. 
Remark 4.2. By Lemma 4.1, any process satisfying the first four conditions in (4.3) automatically satisfies the state constraint $x_{1}(t) \geq 0$ for all $t \in\left[t_{0}, T\right]$. Thus, the latter can be omitted in the problem formulation. This means that, for the case $\alpha=1$, instead of the maximum principle in Theorem 3.4 one can apply the maximum principle for optimal control problems without state constraints in [30, Theorem 6.2.1] (see also [14, Proposition 2.5]). Note that both theorems yield the same necessary optimality conditions (see, e.g., [14, Subsection 3.2]).

Remark 4.3. For the case $\alpha \in(0,1)$, one cannot claim that any process satisfying the first four conditions in 4.3 automatically satisfies the state constraint $x_{1}(t) \geq 0$ for all $t \in\left[t_{0}, T\right]$. Thus, if we consider problem $\left(\mathrm{GP}_{1 a}\right)$ under the conditions (A2) and (B1), or (A2) and (B2), then we have to rely on Theorem 3.4. Referring to the classification of optimal economic growth models given in [13, Section 4], we can say that models of the types "Nonlinear-linear" and "Nonlinear-nonlinear" may require the use of Theorem 3.4. For this reason, we prefer to present the latter in this paper to prepare a suitable framework for dealing with $\left(\mathrm{GP}_{1 a}\right)$ under different sets of assumptions.

Recall that $(\bar{x}, \bar{s})$ is a $W^{1,1}$ local minimizer for $\left(\mathrm{GP}_{1 a}\right)$. It is easy to show that, for any $\delta>0$, there are constants $M_{1}>0$ and $M_{2}>0$ such that $k(t, x):=M_{1}+M_{2} e^{-\lambda t}$ satisfies the conditions described in the hypothesis (H1) of Theorem 3.4. The fulfillment of the hypotheses (H2)-(H4) is obvious. Applying Theorem 3.4, we can find $p \in W^{1,1}\left(\left[t_{0}, T\right] ; \mathbb{R}^{2}\right)$, $\gamma \geq 0, \mu \in C^{\oplus}\left(t_{0}, T\right)$, and a Borel measurable function $\nu:\left[t_{0}, T\right] \rightarrow \mathbb{R}^{2}$ such that $(p, \mu, \gamma) \neq$ $(0,0,0)$, and for $q(t):=p(t)+\eta(t)$ with

$$
\eta(t):=\int_{\left[t_{0}, t\right)} \nu(\tau) d \mu(\tau), \quad \forall t \in\left[t_{0}, T\right)
$$

and

$$
\eta(T):=\int_{\left[t_{0}, T\right]} \nu(\tau) d \mu(\tau)
$$

conditions (i)-(iv) in Theorem 3.4 hold true.

Let us expose the meanings of the conditions (i)-(iv) in Theorem 3.4 .

Condition (i). Note that

$$
\begin{aligned}
& \mu\left\{t \in\left[t_{0}, T\right] \mid \nu(t) \notin \partial_{x}^{>} h(t, \bar{x}(t))\right\} \\
= & \mu\left\{t \in\left[t_{0}, T\right] \mid \partial_{x}^{>} h(t, \bar{x}(t))=\emptyset\right\}+\mu\left\{t \in\left[t_{0}, T\right] \mid \partial_{x}^{>} h(t, \bar{x}(t)) \neq \emptyset, \nu(t) \notin \partial_{x}^{>} h(t, \bar{x}(t))\right\} .
\end{aligned}
$$

Since $\bar{x}_{1}(t) \geq 0$ for every $t$, combining this with 4.7 gives

$$
\begin{aligned}
& \mu\left\{t \in\left[t_{0}, T\right] \mid \nu(t) \notin \partial_{x}^{>} h(t, \bar{x}(t))\right\} \\
= & \mu\left\{t \in\left[t_{0}, T\right] \mid \bar{x}_{1}(t)>0\right\}+\mu\left\{t \in\left[t_{0}, T\right] \mid \bar{x}_{1}(t)=0, \nu(t) \neq(-1,0)\right\} .
\end{aligned}
$$


So, from (i) it follows that

$$
\mu\left\{t \in\left[t_{0}, T\right] \mid \bar{x}_{1}(t)>0\right\}=0
$$

and $\mu\left\{t \in\left[t_{0}, T\right] \mid \bar{x}_{1}(t)=0, \nu(t) \neq(-1,0)\right\}=0$.

Condition (ii). By 4.6), (ii) implies that

$$
-\dot{p}(t)=\left((A \bar{s}(t)-\sigma) q_{1}(t)+(\bar{s}(t)-1) e^{-\lambda t} q_{2}(t), 0\right) \quad \text { a.e. } t \in\left[t_{0}, T\right] .
$$

Hence, $p_{2}(t)$ is a constant for all $t \in\left[t_{0}, T\right]$ and

$$
\dot{p}_{1}(t)=-(A \bar{s}(t)-\sigma) q_{1}(t)+(1-\bar{s}(t)) e^{-\lambda t} q_{2}(t) \quad \text { a.e. } t \in\left[t_{0}, T\right] .
$$

Condition (iii). By the formulas for $g$ and $C, \partial g\left(\bar{x}\left(t_{0}\right), \bar{x}(T)\right)=\{(0,0,0,1)\}$ and $N_{C}\left(\bar{x}\left(t_{0}\right), \bar{x}(T)\right)=\mathbb{R}^{2} \times\{(0,0)\}$. Thus, (iii) yields

$$
\left(p\left(t_{0}\right),-q(T)\right) \in\{(0,0,0, \gamma)\}+\mathbb{R}^{2} \times\{(0,0)\},
$$

which means that $q_{1}(T)=0$ and $q_{2}(T)=-\gamma$.

Condition (iv). By 4.5), from (iv) one gets

$$
\begin{aligned}
& (A \bar{s}(t)-\sigma) \bar{x}_{1}(t) q_{1}(t)+(\bar{s}(t)-1) \bar{x}_{1}(t) e^{-\lambda t} q_{2}(t) \\
= & \max _{s \in[0,1]}\left\{(A s-\sigma) \bar{x}_{1}(t) q_{1}(t)+(s-1) \bar{x}_{1}(t) e^{-\lambda t} q_{2}(t)\right\}
\end{aligned}
$$

for almost every $t \in\left[t_{0}, T\right]$. Equivalently, we have

$$
\left(A q_{1}(t)+e^{-\lambda t} q_{2}(t)\right) \bar{x}_{1}(t) \bar{s}(t)=\max _{s \in[0,1]}\left\{\left(A q_{1}(t)+e^{-\lambda t} q_{2}(t)\right) \bar{x}_{1}(t) s\right\} \quad \text { a.e. } t \in\left[t_{0}, T\right] .
$$

Since $\bar{x}_{1}(t)>0$ for all $t \in\left[t_{0}, T\right]$, it follows that

$$
\left(A q_{1}(t)+e^{-\lambda t} q_{2}(t)\right) \bar{s}(t)=\max _{s \in[0,1]}\left\{\left(A q_{1}(t)+e^{-\lambda t} q_{2}(t)\right) s\right\} \quad \text { a.e. } t \in\left[t_{0}, T\right] .
$$

To prove that the optimal control problem in question has a unique optimal solution under a mild condition imposed on the data tube $(A, \sigma, \lambda)$, we have to deepen the above analysis of the conditions (i)-(iv). As $\bar{x}_{1}(t)>0$ for all $t \in\left[t_{0}, T\right]$ by Lemma 4.1, the equality 4.14 implies that $\mu\left(\left[t_{0}, T\right]\right)=0$, i.e., $\mu=0$. Combining this with 4.12 and (4.13), one gets $\eta(t)=0$ for all $t \in\left[t_{0}, T\right]$. Thus, the relation $q(t)=p(t)+\eta(t)$ allows us to have $q(t)=p(t)$ for every $t \in\left[t_{0}, T\right]$. Therefore, the properties of $p(t)$ and $q(t)$ established in the above analysis of the conditions (ii) and (iii) imply that $p_{2}(t)=-\gamma$ for every $t \in\left[t_{0}, T\right], p_{1}(T)=0$, and

$$
\dot{p}_{1}(t)=-(A \bar{s}(t)-\sigma) p_{1}(t)+\gamma(\bar{s}(t)-1) e^{-\lambda t} \quad \text { a.e. } t \in\left[t_{0}, T\right] .
$$


Now, by substituting $q_{1}(t)=p_{1}(t)$ and $q_{2}(t)=-\gamma$ into 4.15), we have

$$
\left(A p_{1}(t)-\gamma e^{-\lambda t}\right) \bar{s}(t)=\max _{s \in[0,1]}\left\{\left(A p_{1}(t)-\gamma e^{-\lambda t}\right) s\right\} \quad \text { a.e. } t \in\left[t_{0}, T\right] .
$$

Describing the adjoint trajectory corresponding to the control function $\bar{s}(\cdot)$, next lemma is an analogue of Lemma 4.1 .

Lemma 4.4. The Cauchy problem defined by the differential equation (4.16) and the condition $p_{1}(T)=0$ possesses a unique solution $p_{1}(\cdot):\left[t_{0}, T\right] \rightarrow \mathbb{R}$,

$$
p_{1}(t)=-\int_{t}^{T} c(z) \bar{\Omega}(z, t) d z, \quad \forall t \in\left[t_{0}, T\right],
$$

where $\bar{\Omega}(t, \tau)$ is defined by 4.10 for $s(t)=\bar{s}(t)$, i.e.,

$$
\bar{\Omega}(t, \tau):=e^{\int_{\tau}^{t}(A \bar{s}(z)-\sigma) d z}, \quad \forall t, \tau \in\left[t_{0}, T\right]
$$

and

$$
c(t):=\gamma(\bar{s}(t)-1) e^{-\lambda t}, \quad \forall t \in\left[t_{0}, T\right] .
$$

In addition, for any fixed value $\tau \in\left[t_{0}, T\right]$, one has

$$
p_{1}(t)=p_{1}(\tau) \bar{\Omega}(\tau, t)-\int_{t}^{\tau} c(z) \bar{\Omega}(z, t) d z, \quad \forall t \in\left[t_{0}, T\right] .
$$

Proof. Since $\bar{s}(\cdot)$ is measurable and bounded, the function $t \mapsto c(t)$ defined by 4.20 is also measurable and bounded on $\left[t_{0}, T\right]$. Moreover, the function $t \mapsto A \bar{s}(t)-\sigma$ is also measurable and bounded on $\left[t_{0}, T\right]$. In particular, both functions $c(\cdot)$ and $A \bar{s}(\cdot)-\sigma$ are Lebesgue integrable on $\left[t_{0}, T\right]$. Hence, by the lemma in [2, pp. 121-122] we can assert that, for any $\tau \in\left[t_{0}, T\right]$ and $\eta \in \mathbb{R}$, the Cauchy problem defined by the linear differential equation (4.16) and the initial condition $p_{1}(\tau)=\eta$ has a unique solution $p_{1}(\cdot):\left[t_{0}, T\right] \rightarrow \mathbb{R}$. As shown in the proof of Lemma $4.1, \bar{\Omega}(t, \tau)$ given in 4.19$)$ is the principal solution of the homogeneous equation

$$
\dot{\bar{x}}_{1}(t)=(A \bar{s}(t)-\sigma) \bar{x}_{1}(t) \quad \text { a.e. } t \in\left[t_{0}, T\right] .
$$

Besides, by the form of (4.16) and by the theorem in [2, p. 123], the solution of 4.16 is given by 4.21). Especially, applying this formula for the case $\tau=T$ and note that $p_{1}(T)=0$, we obtain 4.18.

In Theorem 3.4, the objective function $g$ plays a role in condition (iii) only if $\gamma>0$. In such a situation, the maximum principle is said to be normal. Investigations on the normality of maximum principles for optimal control problems are available in 9 [1]. For the problem $\left(\mathrm{GP}_{1 a}\right)$, by using $4.18-4.20$ and the property $(p, \mu, \gamma) \neq(0,0,0)$, we now show that the situation $\gamma=0$ cannot happen. 
Lemma 4.5. One must have $\gamma>0$.

Proof. Suppose on the contrary that $\gamma=0$. Then, $c(t) \equiv 0$ by 4.20. Hence, from 4.18) it follows that $p_{1}(t) \equiv 0$. Combining this with the facts that $p_{2}(t)=-\gamma=0$ for all $t \in\left[t_{0}, T\right]$ and $\mu=0$, we get a contradiction to the requirement $(p, \mu, \gamma) \neq(0,0,0)$ in Theorem 3.4 .

To define the control value $\bar{s}(t)$ by $(4.17)$, it is important to know the sign of the real-valued function

$$
\psi(t):=A p_{1}(t)-\gamma e^{-\lambda t}
$$

for each $t \in\left[t_{0}, T\right]$. Namely, one has $\bar{s}(t)=1$ whenever $\psi(t)>0$ and $\bar{s}(t)=0$ whenever $\psi(t)<0$. Hence $\bar{s}(\cdot)$ is a constant function on each segment where $\psi(\cdot)$ has a fixed sign. The forthcoming lemma gives formulas for $\bar{x}_{1}(\cdot)$ and $p_{1}(\cdot)$ on such a segment.

Lemma 4.6. Let $\left[t_{1}, t_{2}\right] \subset\left[t_{0}, T\right]$ and $\tau \in\left[t_{1}, t_{2}\right]$ be given arbitrarily.

(a) If $\bar{s}(t)=1$ for a.e. $t \in\left[t_{1}, t_{2}\right]$, then

$$
\bar{x}_{1}(t)=\bar{x}_{1}(\tau) e^{(A-\sigma)(t-\tau)}, \quad \forall t \in\left[t_{1}, t_{2}\right]
$$

and

$$
p_{1}(t)=p_{1}(\tau) e^{-(A-\sigma)(t-\tau)}, \quad \forall t \in\left[t_{1}, t_{2}\right] .
$$

(b) If $\bar{s}(t)=0$ for a.e. $t \in\left[t_{1}, t_{2}\right]$, then

$$
\bar{x}_{1}(t)=\bar{x}_{1}(\tau) e^{-\sigma(t-\tau)}, \quad \forall t \in\left[t_{1}, t_{2}\right]
$$

and

$$
p_{1}(t)=p_{1}(\tau) e^{\sigma(t-\tau)}+\frac{\gamma}{\sigma+\lambda} e^{\sigma t}\left[e^{-(\sigma+\lambda) t}-e^{-(\sigma+\lambda) \tau}\right], \quad \forall t \in\left[t_{1}, t_{2}\right]
$$

Proof. If $\bar{s}(t)=1$ for a.e. $t \in\left[t_{1}, t_{2}\right]$, then 4.23 is obtained from 4.8 with $x_{1}(\cdot)=\bar{x}_{1}(\cdot)$ and $s(\cdot)=\bar{s}(\cdot)$. Besides, as $\bar{s}(\cdot) \equiv 1$ a.e. on $\left[t_{1}, t_{2}\right]$, the function $c(t)$ defined in 4.20 equals 0 a.e. on $\left[t_{1}, t_{2}\right]$, which implies that the integral in (4.21) vanishes. In addition, substituting the formulas for $\bar{s}(\cdot)$ and $\bar{x}_{1}(\cdot)$ on $\left[t_{1}, t_{2}\right]$ to 4.19$)$, we get $\bar{\Omega}(\tau, t)=e^{-(A-\sigma)(t-\tau)}$ for all $t \in\left[t_{1}, t_{2}\right]$. Thus, 4.24 follows from 4.21.

If $\bar{s}(t)=0$ for a.e. $t \in\left[t_{1}, t_{2}\right]$, then we get 4.25) by applying (4.8) with $x_{1}(\cdot)=\bar{x}_{1}(\cdot)$ and $s(\cdot)=\bar{s}(\cdot)$. To prove 4.26, we use 4.21 and the formulas for $\bar{s}(\cdot)$ and $\bar{x}_{1}(\cdot)$ on 
$\left[t_{1}, t_{2}\right]$. Namely, we have $\bar{\Omega}(\tau, t)=e^{\sigma(t-\tau)}, \bar{\Omega}(z, t)=e^{\sigma(t-z)}$, and $c(z)=-\gamma e^{-\lambda z}$ for all $t, z \in\left[t_{1}, t_{2}\right]$. Substituting these formulas to 4.21 yields

$$
\begin{aligned}
p_{1}(t) & =p_{1}(\tau) e^{\sigma(t-\tau)}-\int_{t}^{\tau}\left(-\gamma e^{-\lambda z}\right)\left(e^{\sigma(t-z)}\right) d z \\
& =p_{1}(\tau) e^{\sigma(t-\tau)}+\gamma e^{\sigma t} \int_{t}^{\tau} e^{-(\sigma+\lambda) z} d z \\
& =p_{1}(\tau) e^{\sigma(t-\tau)}+\frac{\gamma}{\sigma+\lambda} e^{\sigma t}\left[e^{-(\sigma+\lambda) t}-e^{-(\sigma+\lambda) \tau}\right]
\end{aligned}
$$

for all $t \in\left[t_{1}, t_{2}\right]$. This shows that 4.26 is valid.

To proceed furthermore, put $\Gamma=\left\{t \in\left[t_{0}, T\right] \mid \psi(t)=0\right\}$. Since the functions $p_{1}(\cdot)$ is absolutely continuous on $\left[t_{0}, T\right]$, so is $\psi(\cdot)$. Thus, the set $\Gamma$ is compact. Besides, as $p_{1}(T)=0$ and $\gamma>0$, the equality $\psi(T)=A p_{1}(T)-\gamma e^{-\lambda T}$ implies that $\psi(T)<0$. Thus, $T \notin \Gamma$.

First, consider the situation where $\Gamma=\emptyset$. Then we have $\psi(t)<0$ on the whole segment $\left[t_{0}, T\right]$. Indeed, otherwise we would find a point $\tau \in\left[t_{0}, T\right)$ such that $\psi(\tau)>0$. Since $\psi(\tau) \psi(T)<0$, by the continuity of $\psi(\cdot)$ on $\left[t_{0}, T\right]$ we can assert that $\Gamma \cap(\tau, T) \neq \emptyset$. This contradicts our assumption that $\Gamma=\emptyset$. Now, as $\psi(t)<0$ for all $t \in\left[t_{0}, T\right]$, from (4.17) we have $\bar{s}(t)=0$ for a.e. $t \in\left[t_{0}, T\right]$. Applying Lemma 4.6 for $t_{1}=t_{0}, t_{2}=T$, and $\tau=t_{0}$, we get $\bar{x}_{1}(t)=k_{0} e^{-\sigma\left(t-t_{0}\right)}$ for all $t \in\left[t_{0}, T\right]$.

Now, consider the situation where $\Gamma \neq \emptyset$. Let

$$
\alpha_{1}:=\min \{t \mid t \in \Gamma\} \quad \text { and } \quad \alpha_{2}:=\max \{t \mid t \in \Gamma\}
$$

Since $\psi(T)<0$, we see that $t_{0} \leq \alpha_{1} \leq \alpha_{2}<T$. Moreover, by the continuity of $\psi(\cdot)$, and the fact that $\psi(T)<0$, we have $\psi(t)<0$ for every $t \in\left(\alpha_{2}, T\right]$. This and 4.17) imply that $\bar{s}(t)=0$ for almost every $t \in\left[\alpha_{2}, T\right]$. Invoking Lemma 4.6 for $t_{1}=\alpha_{2}, t_{2}=T$, and $\tau=\alpha_{2}$, we obtain $\bar{x}_{1}(t)=\bar{x}_{1}\left(\alpha_{2}\right) e^{-\sigma\left(t-\alpha_{2}\right)}$ for all $t \in\left[\alpha_{2}, T\right]$. If $t_{0}<\alpha_{1}$, then to find $\bar{s}(\cdot)$ and $\bar{x}_{1}(\cdot)$ on $\left[t_{0}, \alpha_{1}\right]$, we will use the following observation.

Lemma 4.7. Suppose that $t_{0}<\alpha_{1}$. If $\psi\left(t_{0}\right)<0$, then $\bar{s}(t)=0$ for a.e. $t \in\left[t_{0}, \alpha_{1}\right]$ and $\bar{x}_{1}(t)=k_{0} e^{-\sigma\left(t-t_{0}\right)}$ for all $t \in\left[t_{0}, \alpha_{1}\right]$. If $\psi\left(t_{0}\right)>0$, then $\bar{s}(t)=1$ for a.e. $t \in\left[t_{0}, \alpha_{1}\right]$ and $\bar{x}_{1}(t)=k_{0} e^{(A-\sigma)\left(t-t_{0}\right)}$ for all $t \in\left[t_{0}, \alpha_{1}\right]$.

Proof. From the condition $t_{0}<\alpha_{1}$ it follows that $\psi\left(t_{0}\right) \psi(t)>0$ for all $t \in\left[t_{0}, \alpha_{1}\right)$. Indeed, otherwise there exists $\tau \in\left(t_{0}, \alpha_{1}\right)$ satisfying $\psi\left(t_{0}\right) \psi(\tau)<0$, which together with the continuity of $\psi(\cdot)$ implies that there is some $\bar{t} \in \Gamma$ with $\bar{t}<\alpha_{1}$. This contradicts the definition of $\alpha_{1}$. If $\psi\left(t_{0}\right)<0$, then $\psi(t)<0$ for all $t \in\left[t_{0}, \alpha_{1}\right)$. Hence, by $(4.17), \bar{s}(t)=0$ for a.e. $t \in\left[t_{0}, \alpha_{1}\right]$. If $\psi\left(t_{0}\right)>0$, then $\psi(t)>0$ for all $t \in\left[t_{0}, \alpha_{1}\right)$. In this situation, by (4.17) we have $\bar{s}(t)=1$ for a.e. $t \in\left[t_{0}, \alpha_{1}\right]$. Thus, in both situations, applying Lemma 4.6 for $t_{1}=t_{0}, t_{2}=\alpha_{1}$, and $\tau=t_{0}$, we obtain the desired formulas for $\bar{x}_{1}(\cdot)$ on $\left[t_{0}, \alpha_{1}\right]$. 
If $\alpha_{1} \neq \alpha_{2}$, then we must have a complete understanding of the behavior of the function $\psi(t)$ on the whole interval $\left[\alpha_{1}, \alpha_{2}\right]$. Towards that aim, we are going to establish three lemmas.

Lemma 4.8. There does not exist any subinterval $\left[t_{1}, t_{2}\right]$ of $\left[t_{0}, T\right]$ with $t_{1}<t_{2}$ such that $\psi\left(t_{1}\right)=\psi\left(t_{2}\right)=0$, and $\psi(t)>0$ for every $t \in\left(t_{1}, t_{2}\right)$.

Proof. On the contrary, suppose that there is a subinterval $\left[t_{1}, t_{2}\right]$ of $\left[t_{0}, T\right]$ with $t_{1}<t_{2}$ such that $\psi(t)>0$ for all $t \in\left(t_{1}, t_{2}\right)$ and $\psi\left(t_{1}\right)=\psi\left(t_{2}\right)=0$. Then, by (4.17) we have $\bar{s}(t)=1$ almost everywhere on $\left[t_{1}, t_{2}\right]$. So, using Lemma 4.6(a) with $\tau=t_{1}$, we have $p_{1}(t)=p_{1}\left(t_{1}\right) e^{-(A-\sigma)\left(t-t_{1}\right)}$ for all $t \in\left[t_{1}, t_{2}\right]$. The condition $\psi\left(t_{1}\right)=0$ implies that $p_{1}\left(t_{1}\right)=\frac{\gamma}{A} e^{-\lambda t_{1}}$. Thus, $p_{1}(t)=\frac{\gamma}{A} e^{-\lambda t_{1}} e^{-(A-\sigma)\left(t-t_{1}\right)}$ for all $t \in\left[t_{1}, t_{2}\right]$. As $\gamma e^{-\lambda t}>0$ for all $t \in\left[t_{0}, T\right]$, the function $\psi_{1}(t):=\frac{\psi(t)}{\gamma e^{-\lambda t}}$ is well defined on $\left[t_{1}, t_{2}\right]$. By the definition of $\psi(\cdot)$, the above formulas for $\bar{x}_{1}(\cdot)$ and $p_{1}(\cdot)$ on $\left[t_{1}, t_{2}\right]$, we have

$$
\psi_{1}(t)=\frac{A p_{1}(t)}{\gamma e^{-\lambda t}}-1=\frac{\gamma e^{-\lambda t_{1}} e^{-(A-\sigma)\left(t-t_{1}\right)}}{\gamma e^{-\lambda t}}-1=e^{(\sigma+\lambda-A)\left(t-t_{1}\right)}-1
$$

for all $t \in\left[t_{1}, t_{2}\right]$. If $\sigma+\lambda-A \neq 0$, then it is easy to see that the equation $\psi_{1}(t)=0$ has a unique solution $t_{1}$ on $\left[t_{1}, t_{2}\right]$. Hence $\psi\left(t_{2}\right) \neq 0$, and we have arrived at a contradiction. If $\sigma+\lambda-A=0$, then $\psi_{1}(t)=0$ for every $t \in\left(t_{1}, t_{2}\right)$. This implies that $\psi(t)=0$ for every $t \in\left(t_{1}, t_{2}\right)$. The latter contradicts our assumption on $\psi(t)$.

The proof is complete.

Lemma 4.9. There does not exist a subinterval $\left[t_{1}, t_{2}\right]$ of $\left[t_{0}, T\right]$ with $t_{1}<t_{2}$ such that $\psi\left(t_{1}\right)=\psi\left(t_{2}\right)=0$ and $\psi(t)<0$ for all $t \in\left(t_{1}, t_{2}\right)$.

Proof. To argue by contradiction, suppose that there is a subinterval $\left[t_{1}, t_{2}\right]$ of $\left[t_{0}, T\right]$ with $t_{1}<t_{2}, \psi(t)<0$ for all $t \in\left(t_{1}, t_{2}\right)$, and $\psi\left(t_{1}\right)=\psi\left(t_{2}\right)=0$. Then, by 4.17) we have $\bar{s}(t)=0$ almost everywhere on $\left[t_{1}, t_{2}\right]$. Therefore, using Lemma 4.6(b) with $\tau=t_{1}$, we obtain

$$
p_{1}(t)=p_{1}\left(t_{1}\right) e^{\sigma\left(t-t_{1}\right)}+\frac{\gamma}{\sigma+\lambda} e^{\sigma t}\left[e^{-(\sigma+\lambda) t}-e^{-(\sigma+\lambda) t_{1}}\right], \quad \forall t \in\left[t_{1}, t_{2}\right]
$$

The assumption $\psi\left(t_{1}\right)=0$ yields $p_{1}\left(t_{1}\right)=\frac{\gamma}{A} e^{-\lambda t_{1}}$. Thus,

$$
p_{1}(t)=\frac{\gamma}{A} e^{-\lambda t_{1}} e^{\sigma\left(t-t_{1}\right)}+\frac{\gamma}{\sigma+\lambda} e^{\sigma t}\left[e^{-(\sigma+\lambda) t}-e^{-(\sigma+\lambda) t_{1}}\right], \quad \forall t \in\left[t_{1}, t_{2}\right] .
$$

By the definition of $\psi(\cdot)$ and the formulas for $\bar{x}_{1}(\cdot)$ and $p_{1}(\cdot)$ on $\left[t_{1}, t_{2}\right]$, we have

$$
\psi(t)=\gamma e^{-\lambda t_{1}} e^{\sigma\left(t-t_{1}\right)}+\frac{A \gamma}{\sigma+\lambda} e^{\sigma t}\left[e^{-(\sigma+\lambda) t}-e^{-(\sigma+\lambda) t_{1}}\right]-\gamma e^{-\lambda t}, \quad \forall t \in\left[t_{1}, t_{2}\right] .
$$


Consider the function $\psi_{2}(t):=\frac{\psi(t)}{\gamma e^{\sigma t}}$, which is well defined for every $t \in\left[t_{1}, t_{2}\right]$. Then, by an elementary calculation one has

$$
\psi_{2}(t)=\left(\frac{A}{\sigma+\lambda}-1\right)\left[e^{-(\sigma+\lambda) t}-e^{-(\sigma+\lambda) t_{1}}\right], \quad \forall t \in\left[t_{1}, t_{2}\right] .
$$

If $\frac{A}{\sigma+\lambda}-1=0$, then $\psi_{2}(t)=0$ for all $t \in\left[t_{1}, t_{2}\right]$. This yields $\psi(t)=0$ for all $t \in\left[t_{1}, t_{2}\right]$, a contradiction to our assumption that $\psi(t)<0$ for all $t \in\left(t_{1}, t_{2}\right)$. If $\frac{A}{\sigma+\lambda}-1 \neq 0$, then by (4.28) one can assert that $\psi_{2}(t)=0$ if and only if $t=t_{1}$. Equivalently, $\psi(t)=0$ if and only if $t=t_{1}$. The latter contradicts the conditions $\psi\left(t_{2}\right)=0$ and $t_{2} \neq t_{1}$.

Lemma 4.10. If the condition

$$
A \neq \sigma+\lambda
$$

is fulfilled, then we cannot have $\psi(t)=0$ for all $t$ from an open subinterval $\left(t_{1}, t_{2}\right)$ of $\left[t_{0}, T\right]$ with $t_{1}<t_{2}$.

Proof. Suppose that 4.29 is valid. If the claim is false, then we would find $t_{1}, t_{2} \in\left[t_{0}, T\right]$ with $t_{1}<t_{2}$ such that $\psi(t)=0$ for $t \in\left(t_{1}, t_{2}\right)$. So, from 4.22$)$ it follows that

$$
p_{1}(t)=\frac{\gamma}{A} e^{-\lambda t}, \quad \forall t \in\left(t_{1}, t_{2}\right)
$$

Therefore, one has $\dot{p}_{1}(t)=-\frac{\lambda \gamma}{A} e^{-\lambda t}$ for almost every $t \in\left(t_{1}, t_{2}\right)$. This and 4.16) imply that

$$
-(A \bar{s}(t)-\sigma) p_{1}(t)+\gamma(\bar{s}(t)-1) e^{-\lambda t}=-\frac{\lambda \gamma}{A} e^{-\lambda t} \quad \text { a.e. } t \in\left(t_{1}, t_{2}\right) .
$$

Combining this with 4.30 yields

$$
-(A \bar{s}(t)-\sigma) \frac{\gamma}{A} e^{-\lambda t}+\gamma(\bar{s}(t)-1) e^{-\lambda t}=-\frac{\lambda \gamma}{A} e^{-\lambda t} \quad \text { a.e. } t \in\left(t_{1}, t_{2}\right) .
$$

Since $\gamma>0$, simplifying the last equality yields $A=\sigma+\lambda$. This contradicts to 4.29 .

Under a mild condition, the constants $\alpha_{1}$ and $\alpha_{2}$ defined by 4.27) coincide. Namely, the following statement holds true.

Lemma 4.11. If 4.29 is fulfilled, then the situation $\alpha_{1} \neq \alpha_{2}$ cannot occur.

Proof. Suppose on the contrary that 4.29 is satisfied, but $\alpha_{1} \neq \alpha_{2}$. Then, we cannot have $\psi(t)=0$ for all $t \in\left(\alpha_{1}, \alpha_{2}\right)$ by Lemma 4.10. This means that there exists $\bar{t} \in\left(\alpha_{1}, \alpha_{2}\right)$ such that $\psi(\bar{t}) \neq 0$. Put $\bar{\alpha}_{1}=\max \left\{t \in\left[\alpha_{1}, \bar{t}\right] \mid \psi(t)=0\right\}$ and $\bar{\alpha}_{2}=\min \left\{t \in\left[\bar{t}, \alpha_{2}\right] \mid \psi(t)=0\right\}$. It is not hard to see that $\psi\left(\bar{\alpha}_{1}\right)=\psi\left(\bar{\alpha}_{2}\right)=0$ and $\psi(\bar{t}) \psi(t)>0$ for all $t \in\left(\bar{\alpha}_{1}, \bar{\alpha}_{2}\right)$. This is impossible by either Lemma 4.8 when $\psi(\bar{t})>0$ or Lemma 4.9 when $\psi(\bar{t})<0$.

We are now in a position to formulate and prove the main result of this paper. 
Theorem 4.12. Suppose that the assumptions (A1) and (B1) are satisfied. If

$$
A<\sigma+\lambda
$$

then $\left(\mathrm{GP}_{1 a}\right)$ has a unique $W^{1,1}$ local minimizer $(\bar{x}, \bar{s})$, which is a global minimizer, where $\bar{s}(t)=0$ for a.e. $t \in\left[t_{0}, T\right]$ and $\bar{x}_{1}(t)=k_{0} e^{-\sigma\left(t-t_{0}\right)}$ for all $t \in\left[t_{0}, T\right]$. This means that the problem $\left(\mathrm{GP}_{1}\right)$ has a unique solution $(\bar{k}, \bar{s})$, where $\bar{s}(t)=0$ for a.e. $t \in\left[t_{0}, T\right]$ and $\bar{k}(t)=k_{0} e^{-\sigma\left(t-t_{0}\right)}$ for all $t \in\left[t_{0}, T\right]$.

Proof. Suppose that (A1), (B1), and the condition 4.31) are satisfied. According to Theorem 2.1. $\left(\mathrm{GP}_{1}\right)$ has a global solution. Hence $\left(\mathrm{GP}_{1 a}\right)$ also has a global solution.

Let $(\bar{x}, \bar{s})$ be a $W^{1,1}$ local minimizer of $\left(\mathrm{GP}_{1 a}\right)$. As it has already been explained in this section, applying Theorem 3.4 we can find $p \in W^{1,1}\left(\left[t_{0}, T\right] ; \mathbb{R}^{2}\right), \gamma \geq 0, \mu \in$ $C^{\oplus}\left(t_{0}, T\right)$, and a Borel measurable function $\nu:\left[t_{0}, T\right] \rightarrow \mathbb{R}^{2}$ such that $(p, \mu, \gamma) \neq(0,0,0)$ and conditions (i)-(iv) in Theorem 3.4 hold true for $q(t):=p(t)+\eta(t)$ with $\eta(t)$ (resp., $\eta(T)$ ) being given by 4.12 for $t \in\left[t_{0}, T\right)$ (resp., by (4.13)). In the above notations, we consider the set $\Gamma=\left\{t \in\left[t_{0}, T\right] \mid \psi(t)=0\right\}$.

In the case $\Gamma=\emptyset$, we have shown that $\bar{s}(t)=0$ for a.e. $t \in\left[t_{0}, T\right]$ and $\bar{x}_{1}(t)=$ $k_{0} e^{-\sigma\left(t-t_{0}\right)}$ for all $t \in\left[t_{0}, T\right]$ (see the arguments given after Lemma 4.6.).

In the case $\Gamma \neq \emptyset$, we define the numbers $\alpha_{1}$ and $\alpha_{2}$ by (4.27). Thanks to the condition (4.31), which implies 4.29), by Lemma 4.11 we have $\alpha_{2}=\alpha_{1}$. Then, as it was shown before Lemma 4.7 , we must have $\bar{s}(t)=0$ for a.e. $t \in\left[\alpha_{1}, T\right]$ and $\bar{x}_{1}(t)=\bar{x}_{1}\left(\alpha_{1}\right) e^{-\sigma\left(t-\alpha_{1}\right)}$ for all $t \in\left[\alpha_{1}, T\right]$. If $t_{0}=\alpha_{1}$, then we obtain the desired formulas for $\bar{s}(\cdot)$ and $\bar{x}_{1}(\cdot)$.

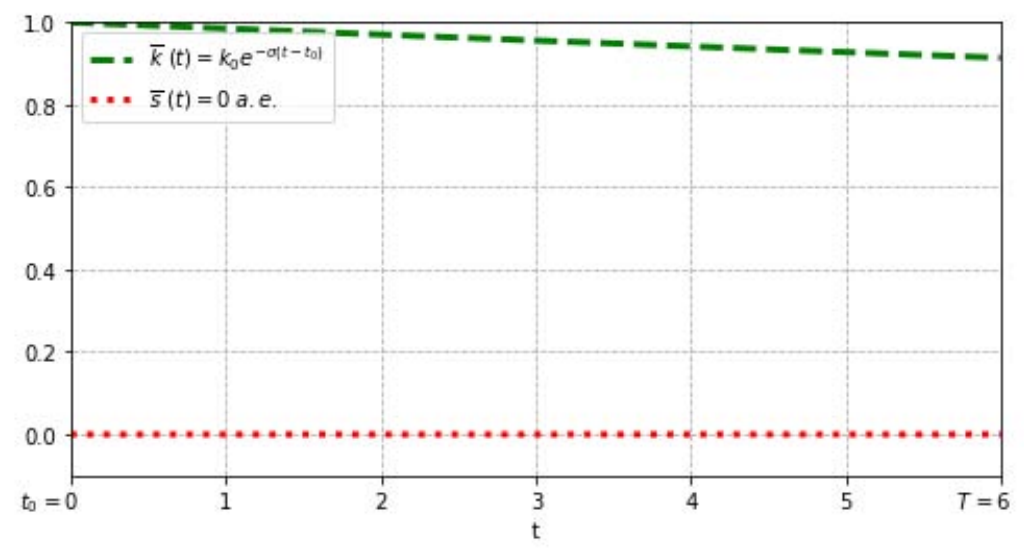

Figure 4.1: The optimal process $(\bar{k}, \bar{s})$ of $\left(\mathrm{GP}_{1}\right)$ corresponding to parameters $\alpha=1, \beta=1$, $A=0.045, \sigma=0.015, \lambda=0.034, k_{0}=1, t_{0}=0$, and $T=6$.

Suppose that $t_{0}<\alpha_{1}$. If $\psi\left(t_{0}\right)<0$, then we can get the desired formulas for $\bar{s}(\cdot)$ and $\bar{x}_{1}(\cdot)$ on $\left[t_{0}, T\right]$ from the formulas for $\bar{s}(\cdot)$ and $\bar{x}_{1}(\cdot)$ on $\left[t_{0}, \alpha_{1}\right]$ in Lemma 4.7 and the just 
mentioned formulas for $\bar{s}(\cdot)$ and $\bar{x}_{1}(\cdot)$ on $\left[\alpha_{1}, T\right]$. If $\psi\left(t_{0}\right)>0$, by Lemma 4.7 one has $\bar{s}(t)=1$ for a.e. $t \in\left[t_{0}, \alpha_{1}\right]$. Then we have

$$
\bar{s}(t)=\left\{\begin{array}{ll}
1 & \text { a.e. } t \in\left[t_{0}, \alpha_{1}\right], \\
0 & \text { a.e. } t \in\left(\alpha_{1}, T\right]
\end{array} \quad \text { and } \quad \bar{x}_{1}(t)= \begin{cases}k_{0} e^{(A-\sigma)\left(t-t_{0}\right)} & \text { if } t \in\left[t_{0}, \alpha_{1}\right] \\
\bar{x}_{1}\left(\alpha_{1}\right) e^{-\sigma\left(t-\alpha_{1}\right)} & \text { if } t \in\left(\alpha_{1}, T\right]\end{cases}\right.
$$

To proceed furthermore, fix an arbitrary number $\varepsilon \in\left(0, \alpha_{1}-t_{0}\right]$ and put $t_{\varepsilon}=\alpha_{1}-\varepsilon$. Consider the control function $s^{\varepsilon}(t)$ defined by setting $s_{\varepsilon}(t)=1$ for all $t \in\left[t_{0}, t_{\varepsilon}\right]$ and $s^{\varepsilon}(t)=0$ for all $t \in\left(t_{\varepsilon}, T\right]$. Denote the trajectory corresponding to $s^{\varepsilon}(\cdot)$ by $x^{\varepsilon}(\cdot)$. Then one has

$$
x_{1}^{\varepsilon}(t)= \begin{cases}k_{0} e^{(A-\sigma)\left(t-t_{0}\right)} & \text { if } t \in\left[t_{0}, t_{\varepsilon}\right] \\ x_{1}^{\varepsilon}\left(t_{\varepsilon}\right) e^{-\sigma\left(t-t_{\varepsilon}\right)} & \text { if } t \in\left(t_{\varepsilon}, T\right] .\end{cases}
$$

Note that

$$
\begin{aligned}
\bar{x}_{2}(T) & =-\int_{t_{0}}^{T}[1-\bar{s}(\tau)] \bar{x}_{1}(\tau) e^{-\lambda \tau} d \tau=-\int_{\alpha_{1}}^{T} \bar{x}_{1}(\tau) e^{-\lambda \tau} d \tau \\
& =-\int_{\alpha_{1}}^{T} \bar{x}_{1}\left(\alpha_{1}\right) e^{-\sigma\left(\tau-\alpha_{1}\right)} e^{-\lambda \tau} d \tau=\frac{\bar{x}_{1}\left(\alpha_{1}\right) e^{\sigma \alpha_{1}}}{\sigma+\lambda}\left[e^{-(\sigma+\lambda) T}-e^{-(\sigma+\lambda) \alpha_{1}}\right] .
\end{aligned}
$$

Since $\bar{x}_{1}\left(\alpha_{1}\right)=k_{0} e^{(A-\sigma)\left(\alpha_{1}-t_{0}\right)}$, it follows that

$$
\bar{x}_{2}(T)=\frac{k_{0}}{\sigma+\lambda} e^{(\sigma-A) t_{0}} e^{A \alpha_{1}}\left[e^{-(\sigma+\lambda) T}-e^{-(\sigma+\lambda) \alpha_{1}}\right] .
$$

Similarly, one gets

$$
x_{2}^{\varepsilon}(T)=\frac{k_{0}}{\sigma+\lambda} e^{(\sigma-A) t_{0}} e^{A t_{\varepsilon}}\left[e^{-(\sigma+\lambda) T}-e^{-(\sigma+\lambda) t_{\varepsilon}}\right] .
$$

Therefore, one gets

$$
\begin{aligned}
& \bar{x}_{2}(T)-x_{2}^{\varepsilon}(T) \\
= & \frac{k_{0} e^{(\sigma-A) t_{0}}}{\sigma+\lambda} \times\left\{e^{A \alpha_{1}}\left[e^{-(\sigma+\lambda) T}-e^{-(\sigma+\lambda) \alpha_{1}}\right]-e^{A t_{\varepsilon}}\left[e^{-(\sigma+\lambda) T}-e^{-(\sigma+\lambda) t_{\varepsilon}}\right]\right\} \\
= & \frac{k_{0} e^{(\sigma-A) t_{0}}}{\sigma+\lambda} \times\left\{e^{-(\sigma+\lambda) T}\left[e^{A \alpha_{1}}-e^{A t_{\varepsilon}}\right]+\left[e^{(A-\sigma-\lambda) t_{\varepsilon}}-e^{(A-\sigma-\lambda) \alpha_{1}}\right]\right\} .
\end{aligned}
$$

Since $t_{\varepsilon} \in\left[t_{0}, \alpha_{1}\right)$, we have $e^{A \alpha_{1}}-e^{A t_{\varepsilon}}>0$. In addition, as $A-\sigma-\lambda<0$ by (4.31), we get $e^{(A-\sigma-\lambda) t_{\varepsilon}}-e^{(A-\sigma-\lambda) \alpha_{1}}>0$. Combining these inequalities with the above expression for $\bar{x}_{2}(T)-x_{2}^{\varepsilon}(T)$, we conclude that $x_{2}^{\varepsilon}(T)<\bar{x}_{2}(T)$. By using (2.1), it is not difficult to show that the norm $\left\|\bar{x}-x^{\varepsilon}\right\|_{W^{1,1}}$ tends to 0 as $\varepsilon$ goes to 0 . So, the inequality $x_{2}^{\varepsilon}(T)<\bar{x}_{2}(T)$, which holds for every $\varepsilon \in\left(0, \alpha_{1}-t_{0}\right]$, implies that the process $(\bar{x}, \bar{s})$ under our consideration cannot be a $W^{1,1}$ local minimizer of $\left(\mathrm{GP}_{1 a}\right)$ (see Definition 3.1). 
Summing up the above analysis and taking into account the fact that $\left(\mathrm{GP}_{1 a}\right)$ has a global minimizer, we can conclude that $\left(\mathrm{GP}_{1 a}\right)$ has a unique $W^{1,1}$ local minimizer $(\bar{x}, \bar{s})$, which is a global minimizer, where $\bar{s}(t)=0$ for a.e. $t \in\left[t_{0}, T\right]$ and $\bar{x}_{1}(t)=k_{0} e^{-\sigma\left(t-t_{0}\right)}$ for all $t \in\left[t_{0}, T\right]$.

It is worthy to state clearly some economic interpretation of Theorem 4.12 .

Remark 4.13. Recall that $\sigma$ is the rate of labor force (closely related to the population growth rate) and $\lambda$ is the real interest rate (which indicates the rate of the decrease along time of the satisfaction level of the society w.r.t. the same amount of consumption). Note that, in the problem $\left(\mathrm{GP}_{1}\right)$, the original production function of the per capita production function $\phi(k)=A k^{\alpha}, k \geq 0$, is given by $F(K, L)=A K^{\alpha} L^{1-\alpha}, K \geq 0, L \geq 0$ (see [13, Section 4]). In the last formula, the exponent $\alpha$ (resp., $1-\alpha$ ) refers to the output elasticity of capital (resp., the output elasticity of labor), which represents the share of the contribution of the capital (resp., of the labor) to the total product $F(K, L)$. Meanwhile, the coefficient $A$ expresses the total factor productivity ${ }^{1}$ (TFP). This measure of economic efficiency is calculated by dividing output by the weighted average of labour and capital input. TFP represents the increase in total production which is in excess of the increase that results from increase in inputs and depends on some intangible factors such as technological change, education, research and development, etc. Therefore, Theorem 4.12 says that if the total factor productivity $A$ is smaller than the sum of the rate of labor force $\sigma$ and the real interest rate $\lambda$, then optimal strategy is to keep the saving equal to 0 . In other words, if the total factor productivity $A$ is relatively small, then an expansion of the production facility does not lead to a higher total consumption satisfaction of the society.

Thanks to the valuable observations and comments from one referee of this paper, we have the following remark.

Remark 4.14. The rate of labor force $\sigma$ is around 1.5\%. The real interest rate $\lambda$ is in general 3.4\%. Hence $\sigma+\lambda=0.049$. Thus, roughly speaking, the assumption $A<\sigma+\lambda$ in Theorem 4.12 means that $A<0.05$. Since weak and very weak economies do exist, the latter assumption is acceptable. Theorem 4.12 is meaningful as here the barrier $A=\sigma+\lambda$ for the TFP appears for the first time. Due to Theorem 4.12, the notions of weak economy (with $A<\sigma+\lambda$ ) and strong economy (with $A>\sigma+\lambda$ ) can have exact meanings. Moreover, the behaviors of a weak economy and of a strong economy might be very different.

By Theorem 4.12 we have solved the problem $\left(\mathrm{GP}_{1}\right)$ in the situation where $A<\sigma+\lambda$. A natural question arises: What happens if $A>\sigma+\lambda$ ? The latter condition means that if the total factor productivity $A$ is relatively large. In this situation, it is likely that the optimal strategy requires to make the maximum saving until a special time $\bar{t} \in\left(t_{0}, T\right)$,

\footnotetext{
${ }^{1}$ See, e.g., https://en.wikipedia.org/wiki/Total_factor_productivity.
} 
which depends on the data tube $(A, \sigma, \lambda)$, then switch the saving to minimum. Further investigations in this direction are going on.

\section{Acknowledgments}

Huong and Yen were supported by National Foundation for Science \& Technology Development (Vietnam) under grant number 101.01-2018.308. Yao was supported by China Medical University, Taichung, Taiwan. The authors are indebted to the anonymous referees for their insightful comments and valuable suggestions.

\section{References}

[1] D. Acemoglu, Introduction to Modern Economic Growth, Princeton University Press, 2009.

[2] V. M. Alekseev, V. M. Tikhomirov and S. V. Fomin, Optimal Control, Consultants Bureau, New York, 1987.

[3] R. J. Barro and X. Sala-i-Martin, Economic Growth, Second edition, MIT Press, 2004 .

[4] D. Cass, Optimum growth in an aggregative model of capital accumulation, Rev. Econ. Stud. 32 (1965), no. 3, 233-240.

[5] L. Cesari, Optimization-Theory and Applications: Problems with ordinary differential equations, Applications of Mathematics (New York) 17, Springer-Verlag, New York, 1983.

[6] A. C. Chiang and K. Wainwright, Fundamental Methods of Mathematical Economics, Fourth edition, McGraw-Hill, 2005.

[7] H. d'Albis, P. Gourdel and C. Le Van, Existence of solutions in continuous-time optimal growth models, Econom. Theory 37 (2008), no. 2, 321-333.

[8] E. D. Domar, Capital expansion, rate of growth, and employment, Econometrica 14 (1946), no. 2, 137-147.

[9] M. M. A. Ferreira and R. B. Vinter, When is the maximum principle for state constrained problems nondegenerate?, J. Math. Anal. Appl. 187 (1994), no. 2, 438-467.

[10] F. A. C. C. Fontes and H. Frankowska, Normality and nondegeneracy for optimal control problems with state constraints, J. Optim. Theory Appl. 166 (2015), no. 1, $115-136$. 
[11] H. Frankowska, Normality of the maximum principle for absolutely continuous solutions to Bolza problems under state constraints, Control Cybernet. 38 (2009), no. 48, $1327-1340$.

[12] R. F. Harrod, An essay in dynamic theory, Econ. J. 49 (1939), no. 193, 14-33.

[13] V. T. Huong, Solution existence theorems for finite horizon optimal economic growth problems, arXiv:2001.03298.

[14] V. T. Huong, J.-C. Yao and N. D. Yen, Analyzing a maximum principle for finite horizon state constrained problems via parametric examples, Part 1: Problems with unilateral state constraints, J. Nonlinear Convex Anal. 21 (2020), no. 1, 157-182.

[15] _ Analyzing a maximum principle for finite horizon state constrained problems via parametric examples, Part 2: Problems with Bilateral state constraints, arXiv:1901.09718.

[16] A. D. Ioffe and V. M. Tihomirov, Theory of Extremal Problems, Studies in Mathematics and its Applications 6, North-Holland, Amsterdam, 1979.

[17] A. N. Kolmogorov and S. V. Fomin, Introductory Real Analysis, Dovers, New York, 1970.

[18] T. C. Koopmans, On the concept of optimal economic growth, in: The Econometric Approach to Development Planning, 225-295, North-Holland, Amsterdam, 1965.

[19] C. Le Van, R. Boucekkine and C. Saglam, Optimal control in infinite horizon problems: a Sobolev space approach, Econom. Theory 32 (2007), no. 3, 497-509.

[20] D. G. Luenberger, Optimization by Vector Space Methods, John Wiley \& Sons, New York, 1969.

[21] B. S. Mordukhovich, Variational Analysis and Generalized Differentiation I: Basic Theory, Grundlehren der Mathematischen Wissenschaften 330, Springer-Verlag, New York, 2006.

[22] — Variational Analysis and Generalized Differentiation II: Applications, Grundlehren der Mathematischen Wissenschaften 331, Springer-Verlag, New York, 2006.

[23] _ Variational Analysis and Applications, Springer Monographs in Mathematics, Springer, Cham, 2018. 
[24] M. S. Nikol'skii, Study of an optimal control problem related to the Solow control model, Proc. Steklov Inst. Math. 292 (2016), suppl. 1, S231-S237.

[25] F. P. Ramsey, A mathematical theory of saving, Econ. J. 38 (1928), no. 152, 543-559.

[26] R. M. Solow, A contribution to the theory of economic growth, Quart. J. Econom. 70 (1956), no. 1, 65-94.

[27] T. W. Swan, Economic growth and capital accumulation, Economic Record 32 (1956), no. $2,334-361$.

[28] A. Takayama, Mathematical Economics, The Dryden Press, Hinsdale, Illinois, 1974.

[29] P. N. V. Tu, Introductory Optimization Dynamics: Optimal control with economics and management science applications, Springer-Verlag, Berlin, 1984.

[30] R. Vinter, Optimal Control, Systems \& Control: Foundations \& Applications, Birkhäuser Boston, Boston, MA, 2000.

Vu Thi Huong

Institute of Mathematics, Vietnam Academy of Science and Technology, 18 Hoang Quoc Viet, Hanoi 10307, Vietnam

E-mail address: vthuong@math.ac.vn, huong263@gmail.com

Jen-Chih Yao

Center for General Education, China Medical University, Taichung 40402, Taiwan

E-mail address: yaojc@mail.cmu.edu.tw

Nguyen Dong Yen

Institute of Mathematics, Vietnam Academy of Science and Technology, 18 Hoang Quoc Viet, Hanoi 10307, Vietnam

E-mail address: ndyen@math.ac.vn 